\title{
Associação entre Transtorno Disfórico Pré-menstrual e Transtornos Depressivos
}

Maria Laura Nogueira Pires $^{1}$ e Helena Maria Calil ${ }^{2}$

Resumo O Transtorno Disfórico Pré-menstrual (TDPM) é caracterizado por uma constelação de sintomas emocionais e alterações comportamentais, que se manifesta com um padrão temporal cíclico em associação com o período pré-menstrual. A validade de uma categoria diagnóstica psiquiátrica distinta para TDPM ainda é uma questão controversa. Entretanto, inúmeros estudos têm documentado uma associação específica entre mudanças prémenstruais e transtornos depressivos. A presente revisão tem como objetivo sintetizar os resultados a respeito da semelhança dos sintomas depressivos no TDPM e nos transtornos depressivos, co-morbidade, história familiar e variáveis biológicas. Evidências de que os sintomas pré-menstruais seriam expressão de vulnerabilidade ao desenvolvimento de estados disfóricos (depressão e ansiedade), assim como as implicações diagnósticas dessa proposta são comentados.

Descritores Disforia pré-menstrual; depressão; ansiedade; co-morbidade; vulnerabilidade

Abstract Premenstrual Dysphoric Disorder (PMDD) is characterised by a myriad of emotional symptoms and behavioural alterations, which occur with a cyclic temporal pattern associated with the pre-menstrual period. The validity of an isolated psychiatric diagnostic category for PMDD remains controversial. However, research results have been showing a specific association between pre-menstrual changes and depressive disorder. The present review has as objective to sintethise the results on similarity between depressive symptoms in PMDD and in the depressive disorders, comorbidity, family history and biological variables. Evidences suggesting that the pre-menstrual symptoms would be expression of a vulnerability to the development of dysphoric states (depression and anxiety) as well as diagnostic implications of such proposals are discussed.

Keywords Pre-menstrual dysphoria; depression; anxiety; comorbidity; vulnerability

\section{Introdução}

A primeira descrição moderna de um conjunto de mudanças no humor e comportamento associadas à fase pré-menstrual é creditada ao ginecologista Robert Frank, que publicou suas observações a respeito do quadro clínico que denominou Tensão Pré-Menstrual: "O grupo de mulheres a que me refiro queixa-se especialmente de um sentimento de tensão indescritível que surge de dez a sete dias antes da menstruação, perdurando em geral até o início do fluxo menstrual. Essas pacientes reclamam de desassossego, irritabilidade, nervos à flor da pele, e procuram alívio através de ações consideradas tolas e destemperadas. Seu sofrimento pessoal é intenso e manifesta-se por muitos atos impetuosos eventualmente repreensíveis (...) Dentro de uma ou duas horas após o início do fluxo menstrual, ocorre um completo alí- vio, tanto da tensão mental quanto física". ${ }^{1}$

Até meados da década de 50, as mulheres com tensão prémenstrual, ou síndrome pré-menstrual (SPM), denominação cunhada por Greene e Dalton, ${ }^{2}$ eram atendidas quase que exclusivamente por profissionais da ginecologia e endocrinologia, e embora comumente apresentassem sintomas psiquiátricos, muito raro chegavam a ser encaminhadas à psiquiatria. ${ }^{3}$

Os aspectos psiquiátricos da síndrome, todavia, foram sendo gradativamente enfatizados e o reconhecimento da síndrome pré-menstrual, na qual as mudanças disfóricas do humor (depressão, irritabilidade, ansiedade) são os sintomas mais marcantes, firmava-se como questão importante dentro do campo da saúde mental. ${ }^{4-6}$

Em 1985, foi constituída uma comissão de assessores da

1. Pesquisadora da Associação Fundo de Incentivo à Psicofarmacologia (Afip) - Departamento de Psicobiologia da Unifesp/EPM. 2. Professora Adjunta, Livre-Docente em Psiquiatria - Departamento de Psicobiologia da Unifesp/EPM. 
Associação Psiquiátrica Americana (APA) com o objetivo de considerar a inclusão, na terceira edição revisada do Manual Diagnóstico e Estatístico - DSM III-R, ${ }^{7}$ de uma categoria diagnóstica para um subgrupo de mudanças pré-menstruais, caracterizado por predomínio de sintomas emocionais e alterações comportamentais, com gravidade clinicamente significativa, e acompanhados de consequiências incapacitantes nas atividades familiar, ocupacional e social. ${ }^{8}$

Contudo, a possibilidade de tal inclusão cercou-se de controvérsia considerável, ${ }^{8,9}$ e a polêmica foi amenizada com a inclusão no apêndice A do DSM III-R de um conjunto de critérios diagnósticos para o quadro agora denominado Transtorno Disfórico da Fase Lútea Tardia (TDFLT). ${ }^{7}$ Nesse apêndice estão as propostas de categorias diagnósticas, as quais necessitam estudos adicionais antes da inserção definitiva no Manual.

Nos anos subseqüentes, grupos de trabalho constituídos pela Associação Psiquiátrica Americana dedicaram-se à revisão de cada categoria diagnóstica do DSM-III-R e elaboração de recomendações a serem incorporadas à quarta edição do Manual de Estatística e Diagnóstico - DSM-IV. ${ }^{10} \mathrm{O}$ grupo responsável pela análise da categoria TDFLT considerou que as evidências ainda eram insuficientes para justificar a sua integração na nomenclatura oficial do DSM-IV, ${ }^{11}$ e concluíram pela permanência dessa categoria no apêndice desse manual, agora renomeada Transtorno Disfórico Pré-Menstrual (TDPM).

Apesar da validade questionável de uma categoria psiquiátrica independente para disforia pré-menstrual, ${ }^{12,13}$ inúmeras evidências se acumulam a respeito de uma associação entre essa condição e transtornos depressivos, levando à sugestão de que um tipo específico de mudanças pré-menstruais, caracterizado por alterações do humor, poderia representar um subtipo de transtorno depressivo, manifestando-se caracteristicamente com um padrão temporal cíclico. ${ }^{14}$

A presente revisão propõe-se a sintetizar as diferentes alternativas que têm sido utilizadas na verificação dessa associação, entre elas a comparação entre mulheres cujas mudanças do humor estão associadas ao período pré-menstrual e aquelas com transtornos de humor no que se refere à apresentação de sintomas, comorbidade, curso, história familiar e variáveis biológicas.

\section{Similaridade dos sintomas depressivos na disforia pré-menstrual e depressão}

Inúmeros investigadores ${ }^{15-17}$ descreveram que a sintomatologia pré-menstrual apresenta-se de maneira muito semelhante à descrita para a depressão atípica: humor deprimido, reativo a eventos favoráveis do ambiente, acompanhado por hipersonia, aumento do apetite com predileção por carboidratos, fadiga, sensibilidade à rejeição, ansiedade e irritabilidade. ${ }^{18}$

Além disso, foi ainda observado que a intensidade da sintomatologia depressiva pré-menstrual é comparativamente mais branda do que a habitualmente encontrada em pacientes com depressão maior. Por exemplo, no estudo de Freeman e col., ${ }^{19}$ a média dos escores da Escala de Hamilton para Depressão (HDRS) ${ }^{20}$ em mulheres $(n=332)$ com Transtorno Disfórico da Fase Lútea Tardia subdivididas de acordo com a faixa etária variou entre $13,5( \pm 5,4)$ e $14,7( \pm 7,2)$ nas mais idosas, valores semelhantes aos descritos por McGrath e col. ${ }^{21}$ para pacientes com depressão atípica $(n=401 ;$ HDRS $=14,4 \pm 3,9)$. A conclusão de que a sintomatologia pré-menstrual é leve ou moderada se repete quando são consideradas as auto-avaliações através do Inventário Beck para Depressão. ${ }^{22}$ Assim, as médias mencionadas em diversas investigações sobre síndrome pré-menstrual ${ }^{23-}$ ${ }^{25}$ variaram entre $14,3( \pm 3,0)$ e $20,1( \pm 4,2)$, índices inferiores aos descritos por Frank e col. ${ }^{26}$ para um grupo de mulheres com diagnóstico de depressão maior pelo "Research Diagnostic Criteria" $(1978 ; n=180 ;$ média $=28.6 \pm 7.6) .{ }^{27}$

Os distúrbios do sono, principalmente hipersonia, durante a fase pré-menstrual, também estão entre os sintomas mais freqüentes em mulheres com o diagnóstico de síndrome prémenstrual. ${ }^{28}$ De fato, Roy-Byrne e col. ${ }^{29}$ descreveram que $71 \%$ da amostra de mulheres com síndrome pré-menstrual prospectivamente confirmada $(\mathrm{n}=59)$ avaliaram como substancial o aumento da necessidade de sono.

Outra característica dos quadros depressivos atípicos comumente observada em mulheres com disforia pré-menstrual diz respeito à oscilação do apetite durante o período prémenstrual, principalmente com um aumento na preferência por carboidratos. ${ }^{28,30}$ Essa predileção por carboidratos no período pré-menstrual tem sido explicada por duas hipóteses principais: 1) Hill e Heaton-Brown, ${ }^{31}$ apoiados nas indicações de que tais alterações ocorrem em mulheres sem patologia psiquiátrica e geralmente não envolvem um tipo específico de alimento, argumentam que elas não estariam necessariamente associadas a flutuações fisiológicas ou do estado psicológico, sendo principalmente motivadas pela fome e pelo bem-estar subjetivo advindos do consumo de alimentos considerados prazerosos; 2) Wurtman (1993) considera que o aumento no consumo de carboidratos, tanto em pacientes com disforia prémenstrual quanto em quadros de depressão atípica, teria uma função compensatória, na medida em que esse consumo é capaz de aumentar a síntese e a liberação de serotonina através do aumento da captação de triptofano, aminoácido precursor da síntese desse neurotransmissor. Dessa maneira, a hipótese de uma associação entre síndrome pré-menstrual e depressão atípica seria fortalecida desde que as alterações no humor e apetite observadas nessas condições pudessem resultar de um desequilíbrio comum a ambas, especificamente do sistema serotonérgico central.

\section{Co-morbidade e curso}

O estudo da associação entre mudanças do humor no período pré-menstrual e transtorno depressivo tem se utilizado da verificação da co-morbidade entre essas condições. Assim, embora com exceção, ${ }^{28}$ há observações de que as freqüências de SPM são superiores entre as pacientes com transtorno depressivo quando comparadas a grupos sem história psiquiátrica. ${ }^{32}$ Também, outros estudos indicam que grande parte das mulheres com SPM apresentam história de transtornos psiquiátricos, especialmente depressão. ${ }^{33}$

No entanto, a comparação entre estimativas acerca da comorbidade de transtornos psiquiátricos durante a vida em 
amostras clínicas deve ser avaliada com cuidado. Regier e col. ${ }^{34}$ comentam que indivíduos com transtornos múltiplos comparecem com maior frequiência a ambientes clínicos à procura de tratamento e dessa forma, as prevalências de co-morbidade entre transtornos psiquiátricos tendem sistematicamente a ser mais elevadas em amostras oriundas de ambientes clínicos do que as observadas na população geral .

Nesse sentido, o estudo de Merikangas e col. ${ }^{35}$ tem importância considerável. Os autores coletaram informações a respeito do vínculo entre sintomas emocionais durante a fase prémenstrual e síndromes psiquiátricas em mulheres participantes do estudo epidemiológico prospectivo realizado com adultos jovens em Zurique, Suíça. Numa análise transversal dos resultados (o período de avaliação compreendia o ano anterior à entrevista, 1988), foram utilizadas as informações de 82 mulheres que relataram presença de sintomas emocionais e somáticos durante a fase pré-menstrual (grupo problemas menstruais) e 136 mulheres que não mencionavam o mesmo (grupo controle). Essa análise mostrou associações significativas entre presença de sintomas emocionais durante a menstruação e diagnóstico de depressão maior realizado de acordo com os critérios do DSM-III (7,4\% do grupo controle apresentou o diagnóstico de depressão maior vs $17,1 \%$ do grupo com problemas menstruais; risco relativo $=2,6)^{36}$ e agorafobia ( $0,7 \%$ do grupo controle vs 7,3\% do grupo com problemas menstruais; risco relativo $=10,7)$. Curiosamente, os grupos não foram discriminados pela presença de outros transtornos ansiosos (transtorno do pânico e ansiedade generalizada, fobia social e simples) nem pelo diagnóstico de transtorno depressivo breve recorrente. Ainda nesse mesmo estudo, uma análise longitudinal utilizou entrevistas realizadas ao longo de 10 anos de investigação (entre 1979 e 1988) com 152 mulheres controles e 147 mulheres com problemas menstruais, demonstrando que o grupo de mulheres com problemas menstruais diferiu do grupo de controles num número ainda maior de síndromes psiquiátricas: depressão maior (23,7\% no grupo controle vs 40,1\% no grupo sintomático; risco relativo $=2,2$ ), transtorno depressivo breve recorrente ( $25,0 \%$ no grupo controle vs 38,1\% no grupo sintomático; risco relativo $=1,8$ ), agorafobia (4,6\% no grupo controle $v s 17,0 \%$ no grupo sintomático; risco relativo $=4,2)$ e fobia simples $(12,9 \%$ no grupo controle $v s 26,9 \%$ no grupo sintomático; risco relativo $=2,5$ ).

$\mathrm{O}$ significado da co-morbidade entre síndrome pré-menstrual e transtorno depressivo também tem sido investigado através do exame da ordem temporal do início dessas condições. No estudo epidemiológico de Zurique, as associações mais freqüentes de síndromes psiquiátricas e dificuldades menstruais observadas longitudinalmente em comparação com a análise transversal foram interpretadas como sugestivas de que a psicopatologia preexistente poderia predispor ao aparecimento de sintomas emocionais associados ao ciclo menstrual. ${ }^{35}$

Outras evidências indicam uma relação temporal contrária, isto é, uma progressão da síndrome pré-menstrual para quadros de depressão maior. ${ }^{37}$ Rubinow e Schmidt $^{38}$ propõem que as mudanças pré-menstruais funcionariam como estímulos sensibilizadores em algumas mulheres, passando a influenci- ar o desenvolvimento, expressão, ou o curso dos episódios depressivos subseqüentes. Dessa maneira, interpretaram o caso de uma paciente de 20 anos na qual puderam estabelecer claramente a ordem temporal dos eventos. ${ }^{39}$ Os episódios depressivos limitados ao período pré-menstrual antecederam os episódios mais duradouros de depressão e, nessas ocasiões, a remissão dos sintomas se deu pela administração de contraceptivo oral.

Assim, a história natural da SPM ainda permanece um assunto controverso. As evidências fragmentam-se entre aquelas sugestivas de uma progressão da síndrome pré-menstrual para um quadro de depressão e outras indicativas de que tenderia à remissão espontânea ou ao abrandamento com o passar do tempo. ${ }^{40}$ Mais ainda, outros investigadores descreveram que a disforia pré-menstrual permanece estável ao longo do tempo, sugerindo uma independência entre essa condição e transtornos depressivos. ${ }^{41}$

A co-morbidade freqüente de transtornos mentais em mulheres com disforia pré-menstrual é um fator complicador no diagnóstico de TDPM. Mais ainda, são várias as evidências de que o período pré-menstrual associa-se com uma maior vulnerabilidade para o agravamento de um transtorno psiquiátrico preexistente. Assim, parte das mulheres que procuram tratamento para síndrome pré-menstrual apresenta uma piora, nesta fase específica do ciclo menstrual, de outros transtornos mentais crônicos, tais como bulimia, distimia, transtorno de ansiedade, abuso de substâncias, entre outros. ${ }^{42}$ Nesse sentido, a análise longitudinal ao longo do ciclo menstrual, através de diários e avaliações semanais tem sido a estratégica recomendada para auxiliar a diferenciação entre a presença de disforia pré-menstrual na ausência de outro transtorno mental atual e exacerbação de condições psicopatológicas preexistentes. Nessa perspectiva, um fator importante para a distinção seria a duração da sintomatologia, ou seja, se o aparecimento e a remissão dos sintomas restringe-se à fase lútea e ao período pós-menstrual, respectivamente.

Contudo, qualquer dessas condições psiquiátricas citadas pode ocorrer concomitantemente ao transtorno disfórico prémenstrual, ${ }^{11}$ e ainda é matéria de estudo o estabelecimento de estratégias que auxiliem a esclarecer se a piora clínica durante a fase lútea representaria uma exacerbação de transtorno preexistente ou uma sobreposição do TDPM a outra condição em curso. $\mathrm{O}$ aparecimento de novos sintomas durante os dias que antecedem a menstruação favoreceria essa última alternativa, embora seja ainda assunto controverso. ${ }^{42}$

\section{Sintomas pré-menstruais e história familiar}

Um volume considerável de evidências levou ao reconhecimento de que os transtornos psiquiátricos agregam-se em famílias, ou seja, há uma prevalência maior de uma determinada psicopatologia entre os familiares de indivíduos afetados em comparação tanto com os familiares de indivíduos não afetados quanto com a prevalência observada na população geral.

Informações a respeito da história psiquiátrica de familiares de pacientes com disforia pré-menstrual são escassas e as que se seguem foram obtidas através do exame das amostras utili- 
zadas em investigações que tinham diferentes propósitos. Harrison e col..$^{43}$ descreveram história familiar de depressão em $73 \%$ da amostra composta por 11 pacientes com TDFLT. Em outra investigação, Harrison e col. ${ }^{44}$ relataram que 28 e $38 \%$ das mulheres controles ( $\mathrm{n}=61)$ e com TDFLT sem história psiquiátrica prévia $(n=86)$ tinham pelo menos um parente de primeiro grau com história de depressão. Esses valores diferiram significantemente do obtido no grupo de pacientes com TDFLT e antecedentes psiquiátricos $(n=86$; principalmente transtorno do humor ou ansioso), no qual história familiar de depressão esteve presente em $62 \%$ da amostra. Além disso, 22 e $28 \%$ das pacientes com TDFLT, que, respectivamente, tinham ou não antecedentes pessoais de história psiquiátrica, referiram que familiares queixavam-se de síndrome pré-menstrual (mãe ou outra parente) mais freqüentemente do que o grupo de mulheres controles $(4 \%)$.

Freeman e col. ${ }^{45}$ observaram que $36 \%$ da amostra de mulheres com síndrome pré-menstrual prospectivamente confirmada $(n=168)$ relatavam que suas mães também eram afetadas com sintomas pré-menstruais. História familiar de transtorno mental (sem especificação) foi referida por aproximadamente metade das pacientes (45\%), sendo que $40 \%$ delas tinham história familiar de alcoolismo.

Entre os primeiros estudos que examinaram de maneira sistemática a presença de um componente familiar para algumas das características do ciclo menstrual destaca-se o de Kantero e Widholm, ${ }^{46}$ os quais estudaram mil pares de mães e filhas e mostraram correlações significativas para inúmeras variáveis, incluindo "tensão pré-menstrual" $(\mathrm{r}=0.24, \mathrm{p}<0.0001)$.

Glick e col. ${ }^{47}$ obtiveram medidas retrospectivas sobre mudanças pré-menstruais para 13 pares de irmãs, todas participantes de um estudo sobre familiares de probandos hospitalizados devido a transtornos afetivos. Segundo esses autores, desde que transtornos afetivos se agregam em famílias e têm sido associados a mudanças disfóricas pré-menstruais, seria plausível inferir que, igualmente, haveria concordância entre as irmãs no que se refere a mudanças pré-menstruais no humor. Os resultados preliminares demonstrativos de concordância para transtornos afetivos confirmaram a premissa: índices significantes de correlação foram obtidos para três das subescalas (humor deprimido, ansiedade e mudanças físicas) derivadas do Pre-menstrual Assesment Form (PAF). ${ }^{16}$ Continuando essa investigação, foi examinada a semelhança dos sintomas pré-menstruais entre 80 pares de irmãs recrutadas da comunidade, utilizando medidas retrospectivas (PAF) e prospectivas longitudinais (Daily Rating Form).$^{48}$ Contrariando as expectativas, embora houvesse concordância para características gerais do ciclo menstrual e aspectos físicos (idade da menarca, duração do ciclo, peso, altura), as irmãs demonstraram pouca concordância tanto para os sintomas físicos quanto os emocionais, em qualquer das medidas utilizadas. Esse resultado se manteve inalterado mesmo quando as irmãs foram subdivididas entre aquelas concordantes ou não quanto à história de transtorno afetivo. Os autores recomendaram que mais estudos sejam conduzidos nessa área, na tentativa de esclarecer, por exemplo, se a concordância seria maior quando as mudanças pré-menstruais se apresentassem com maior gravidade.

Outras investigações apresentam resultados a respeito da concordância quanto ao diagnóstico de síndrome pré-menstrual em gêmeas. Dalton e col. ${ }^{49}$ descrevem que a concordância quanto ao diagnóstico de SPM, prospectivamente avaliado durante um período de três meses, ocorreu em 14 dos 15 (93\%) pares monozigóticos e em 7 dos 16 pares (44\%) dizigóticos.

A agregação familiar para a ocorrência de sintomas prémenstruais também foi descrita por Kendler e col. ${ }^{50}$ e Condon. ${ }^{51}$ Nesse último estudo, as respostas a um questionário retrospectivo para avaliação da síndrome pré-menstrual, desenvolvido por Steiner e col..$^{52}$ foram avaliadas. A correlação encontrada para o escore total no grupo de gêmeas monozigóticas (157 pares; $r=0,55)$ foi significativamente maior do que aquela observada para o grupo de gêmeas dizigóticas (143 pares; $r=$ $0,28)$. Também, todas as correlações obtidas para o grupo de gêmeas monozigóticas nas diversas subescalas excederam as encontradas para as irmãs dizigóticas, sendo que em três delas (tensão, coordenação motora e função cognitiva) as diferenças foram estatisticamente significativas.

\section{Variáveis biológicas envolvidas na disforia pré- menstrual: particularidades e semelhanças com os transtornos depressivos}

Desde a sugestão de Frank ${ }^{1}$ de que a SPM teria causas hormonais, inúmeras tentativas foram feitas com o objetivo de verificar se as mudanças pré-menstruais seriam acompanhadas por alterações no sistema reprodutivo endócrino, principalmente por uma deficiência na produção de progesterona ou desequilíbrio na razão estrógeno/progesterona. ${ }^{2,53}$

Bäckström e col. ${ }^{54}$ investigaram a relação temporal entre as mudanças subjetivas e as hormonais em mulheres nas quais o relato da ciclicidade dos sintomas havia sido confirmada prospectivamente. Para tanto, solicitaram às participantes que anotassem diariamente seus sintomas (depressão e irritabilidade) durante um ciclo menstrual e concomitantemente coletaram sangue para determinação dos níveis plasmáticos dos hormônios ovarianos. Entre os resultados, destaca-se aquele de que em algumas mulheres, o aparecimento dos sintomas coincide com o início da fase lútea, ocasião na qual os níveis de estrógeno e progesterona estão aumentando. $\mathrm{O}$ fato de os sintomas aparecerem antes do declínio hormonal que caracteriza a fase pré-menstrual implica em que esses não poderiam estar sendo provocados, pelo menos unicamente, pela diminuição dos níveis plasmáticos desses hormônios, como antes sugerido. Rubinow e col. ${ }^{55}$ investigaram os níveis plasmáticos e padrão de secreção de vários hormônios, entre eles, progesterona e estradiol, em vários pontos ao longo do ciclo menstrual em grupos de mulheres com síndrome pré-menstrual $(n=17)$ e controles $(n=9)$, e não encontraram diferenças em nenhuma das variáveis estudadas. Similarmente, não foram encontradas diferenças entre as medidas de estrógeno e progesterona, nem na razão entre esses hormônios (E/P) durante as fases sintomáticas ou assintomáticas do ciclo menstrual em oito mulheres com síndrome pré-menstrual 
prospectivamente documentada. ${ }^{56}$

Schmidt e col. ${ }^{57}$ manipularam o ciclo hormonal na fase lútea a fim de correlacionar os eventos dessa fase do ciclo menstrual com o aparecimento dos sintomas pré-menstruais. Em um grupo de mulheres com TDFLT, a fase lútea foi interrompida através da administração, seis dias após o pico do hormônio luteinizante, de um antagonista dos receptores de progesterona (mifepristone, RU-486). Esse agente produz a regressão do corpo lúteo, induzindo a menstruação dentro de 48 a 72 horas. A análise dos resultados mostrou que o bloqueio da ação da progesterona e o término da fase lútea do ciclo menstrual não alteraram o momento de aparecimento e nem a gravidade dos sintomas. $\mathrm{O}$ aparecimento de sintomas durante a fase folicular nesse estudo foi considerado como sugestivo de que os sintomas da síndrome pré-menstrual não são devidos, pelo menos exclusivamente, aos níveis hormonais que caracterizam a fase lútea. Mais ainda, sugeriram que a sintomatologia pré-menstrual poderia estar sendo provocada pelos eventos hormonais que antecedem a fase lútea tardia, de maneira consistente com as demonstrações de que a supressão da ovulação resulta no desaparecimento dos sintomas. ${ }^{58}$ De fato, a principal evidência do envolvimento dos hormônios gonadais na fisiopatologia da disforia pré-menstrual vem de estudos que demonstram redução na gravidade ou desaparecimento dos sintomas após supressão da função ovariana, por exemplo, com o uso de 17 bestradiol ${ }^{59} \mathrm{e}$ análogos do hormônio liberador de gonadotrofinas. ${ }^{60}$

Redei e Freeman ${ }^{61}$ - apoiando-se na sugestão de Schmidt e col.$^{57}$ de que a ovulação seria uma condição necessária para a ocorrência dos sintomas pré-menstruais e que as variações hormonais da fase lútea não seriam responsáveis pela manifestação dos sintomas pré-menstruais - examinaram se os níveis hormonais durante a ovulação e início da fase lútea correlacionar-se-iam com a gravidade da sintomatologia prémenstrual. Os resultados mostraram que os níveis de estrógeno e progesterona, medidos diariamente ao longo do ciclo menstrual, foram consistentemente superiores, porém não de maneira significativa, nas pacientes com síndrome pré-menstrual $(n=10)$, se comparadas ao grupo de mulheres controles $(n=8)$. No entanto, algumas diferenças apareceram quando as pacientes com SPM foram classificadas em dois subgrupos, de acordo com a sintomatologia durante as fases ovulatória e pré-menstrual. Os níveis hormonais daquelas que apresentavam escores baixos em ambas as fases (subgrupo $1 ; n=6$ ) foram semelhantes aos obtidos no grupo controle. Nas restantes (subgrupo $2 ; \mathrm{n}=4$; com sintomatologia mais grave), somente os níveis de progesterona, mas não de estradiol, foram comparáveis aos obtidos no grupo controle. A comparação entre mulheres controles e do subgrupo 2 acerca dos valores de estradiol em cada fase do ciclo menstrual indicaram que para aquelas com sintomas pré-menstruais graves os níveis plasmáticos desse hormônio estiveram significativamente mais elevados entre a fase folicular e início da fase lútea, principalmente por ocasião da ovulação. Esses resultados coincidiram com relato anterior de Hammarback e col., ${ }^{62}$ demonstrando uma correlação significativa entre o aumento dos níveis plasmáticos de estrógeno e progesterona durante as fases periovulatória e lútea e a acentuação da sintomatologia prémenstrual.

A associação entre disforia pré-menstrual e depressão também tem sido examinada em relação às outras alterações hormonais comumente encontradas em pacientes com transtornos depressivos. De maneira geral, a atividade do eixo hipotálamo-hipófise-adrenal tem sido extensivamente estudada na depressão com demonstrações de hipercortisolemia, ausência de supressão da secreção de cortisol após administração de dexametasona e resposta atenuada do ACTH ao teste de estimulação pelo CRH (compatível com hipersecreção de CRH ou ativação do eixo HPA) ${ }^{63}$

Roy-Byrne e col. ${ }^{64}$ realizaram o teste de supressão à dexametasona em 11 mulheres com SPM prospectivamente confirmada e em sete controles. Os resultados não indicaram diferenças significativas entre os grupos nos testes feitos durante as fases folicular ou lútea. Consistentemente, o mesmo grupo de investigadores não encontrou diferenças entre mulheres controles e com síndrome pré-menstrual no padrão de secreção de cortisol pela manhã ao longo do ciclo menstrual. ${ }^{55}$ Em outro estudo, o padrão de secreção de cortisol foi similar entre os grupos controle $(n=16)$ e de mulheres com SPM $(n=16)$, e ambos com valores significativamente menores do que os observados no grupo de mulheres com depressão endógena $(n=6)$. Esses resultados foram interpretados como evidência de que os episódios depressivos no período pré-menstrual diferenciam-se da depressão maior com características endógenas. ${ }^{17}$ Rabin e col ${ }^{65}$ relataram diminuição dos níveis plasmáticos basais de cortisol no período noturno em mulheres com síndrome pré-menstrual comparadas ao grupo controle. Essas diferenças foram observadas tanto na fase folicular quanto lútea, portanto, não estiveram relacionadas ao aparecimento dos sintomas. Mais ainda, as medidas dos níveis urinários de cortisol livre das pacientes com SPM não mostraram diferenças significativas na secreção desse hormônio durante as fases folicular ou lútea, quando comparadas ao padrão das mulheres controles, sugerindo que as mudanças na responsividade do eixo HPA, observadas em mulheres com SPM, são transitórias e breves, não resultando em incremento significativo do cortisol. Ainda nesse estudo, não foram observadas diferenças significativas entre pacientes e controles na resposta do hormônio adrenocorticotrófico (ACTH) ao teste de estimulação pelo hormônio liberador de corticotrofina ovino $(\mathrm{CRH})$ realizado durante as fases folicular e lútea do ciclo menstrual.

Em conjunto, esses resultados, além de sugerirem a integridade do eixo hipotálamo-hipófise-adrenal em pacientes com síndrome pré-menstrual, não corroboram a associação entre essa síndrome e transtorno depressivo maior.

Igualmente, a atividade tireoidiana em pacientes com depressão tem sido objeto de muita pesquisa, mostrando que essa população apresenta resposta atenuada do hormônio estimulante da tireóide (TSH) após estimulação pelo hormônio liberador de tireotropina (TRH). Essa diminuição na secreção do TSH tem sido considerada consequiência de uma hipersecreção crônica do TRH, o qual diminuiria o número de receptores hipofisários 
para TRH. Mais ainda, alguns pacientes com transtornos do humor apresentam níveis elevados de TSH basal ou após estimulação com TRH, condizente com hipotireoidismo. ${ }^{63}$

Assim, Schmidt e col. ${ }^{66}$ examinaram a funcionalidade do eixo hipotálamo-hipófise-tireóide em 124 mulheres com SPM prospectivamente confirmada. Foram encontrados níveis basais alterados de TSH, indicativos de hipo ou hipertireoidismo em $10.5 \%$ da amostra $(n=13)$, percentual comparável ao das prevalências de disfunção tireoidiana em mulheres na idade reprodutiva $(4,6-7,5 \%)$. Além disso, não foram identificadas diferenças entre o grupo de mulheres com síndrome pré-menstrual ( $n=60)$ ou controles $(n=22)$ no padrão de secreção do TSH em resposta à estimulação pelo TRH, confirmando estudos prévios. ${ }^{67,68}$

\section{Envolvimento dos ritmos biológicos na disforia pré-menstrual e depressão}

Um conjunto de evidências indica que uma variedade de ritmos circadianos estariam alterados na depressão, sendo temperatura, cortisol e sono REM ("rapid eyes movement") os mais estudados. Embora não exista consenso, de maneira geral se conclui que a posição de fase de um ou mais ritmos circadianos, tende a um adiantamento em relação ao período do sono. ${ }^{69}$

Várias manipulações experimentais têm procurado definir se a posição de fase dos ritmos circadianos teria um papel causal na sintomatologia depressiva. Nesse sentido, Wehr e col. ${ }^{70}$ propuseram que se um adiantamento de fase de ritmos circadianos em relação ao sono contribui para a manifestação da depressão, então um correspondente adiantamento no período de sono seria capaz de corrigir esse distúrbio interno de fase, levando a uma melhora da sintomatologia depressiva. De fato, acumulam-se evidências documentando que uma noite de privação total de sono, ou mesmo parcial na segunda metade da noite, pode resultar em melhora do humor de pacientes deprimidos. Recentemente, tais evidências têm se estendido para mulheres com disforia pré-menstrual, provendo evidências favoráveis a uma associação entre disforia pré-menstrual e transtornos depressivos..$^{71,72}$

Outras evidências também sugerem que pacientes com SPM apresentam distúrbios de ritmos circadianos. Parry e col. ${ }^{73}$ confirmaram adiantamento de fase em relação ao sono no oscilador circadiano que regula o fim da secreção noturna de melatonina em oito pacientes com SPM, comparadas com mulheres controle. Dando sequiência à investigação, esses investigadores propuseram-se a corrigir o distúrbio circadiano com a utilização de fototerapia (luz de alta intensidade $=2,500$ lux; 2 horas de exposição). Assim, seis pacientes com SPM foram submetidas a fototerapia, administrada pela manhã ou à noite, durante a semana pré-menstrual de dois ciclos menstruais. Esse procedimento adianta ou atrasa os ritmos circadianos, respectivamente. Então, esperava-se que o tratamento noturno resultasse na correção do distúrbio circadiano de adiantamento de fase, implicando também na melhora do humor. Como esperado, os resultados mostraram que somente a fototerapia à noite foi eficaz na redução da sintomatologia depressiva, avaliada pela Escala de Hamilton para Depressão e Inventário Beck para Depressão. Também, mulheres com SPM exibiram um avanço no pico do cortisol durante a fase lútea em comparação com a fase folicular, ao contrário do observado no grupo controle. ${ }^{74}$

A latência para o sono REM, considerada um marcador de ritmos biológicos, também tem sido investigada durante as fases do ciclo menstrual. Lee e col. ${ }^{75}$ relataram uma redução pequena, porém significante, durante a fase lútea, em comparação com a fase folicular, divergindo dos resultados anteriormente obtidos por Parry e col. ${ }^{76}$ Os resultados de Severino ${ }^{77}$ reforçaram a proposição de uma correlação entre posição de fase e estado clínico: as mulheres que apresentaram redução na latência para o sono REM durante a fase pré-menstrual mostraram-se sintomáticas durante o mês de estudo $(n=3)$, ao contrário daquelas cujas medidas foram normais $(n=2)$.

Wehr e Wirz-Justice ${ }^{70}$ (citados em Anderson e Wirz-Justice) ${ }^{69}$ argumentam que a disfunção circadiana não estaria necessariamente envolvida em todos os casos de transtornos do humor, mas sim que deveria ser considerada como um dos fatores entre inúmeros outros. Assim, esse fenômeno somente seria esperado naqueles indivíduos suscetíveis, de tal maneira que a dessincronização interna dos ritmos produziria mudanças no humor apenas entre aqueles geneticamente, biologicamente ou psicologicamente predispostos.

A identificação de possíveis causas de disfunção circadiana, questão ainda pouco investigada em seres humanos, tem sido objeto de pesquisas em animais de laboratório, as quais têm documentado a importância dos esteróides sexuais na regulação dos ritmos biológicos. Leibenluft, ${ }^{78}$ numa revisão, descreveu os resultados da administração de estradiol em fêmeas ovariectomizadas. De maneira geral, ocorre um encurtamento de período dos ritmos circadianos em livre curso e adiantamento no início dos ritmos sincronizados. A administração de estrógeno previne a dessincronização dos ritmos circadianos decorrente da exposição constante à luz. Em fêmeas intactas, o início do período de atividade acontece mais cedo nos dias do ciclo estral que são caracterizados por níveis mais elevados de estradiol.

Também, outras evidências têm comprovado a ação do estrógeno no sistema serotonérgico central, sistema que tem recebido grande atenção e destaque na fisiopatologia dos transtornos do humor e, igualmente, nas mudanças do humor associadas ao ciclo menstrual. ${ }^{79,80}$ Os efeitos desse hormônio são variados e incluem modulação da síntese, taxa de renovação, captação, sítios de ligação e regulação de respostas a agonistas serotonérgicos, geralmente amplificando-as. ${ }^{79,81}$ Mais ainda, o sistema serotonérgico possui projeções ao núcleo supraquiasmático - estrutura cerebral responsável pelo controle do sistema circadiano - evidenciando uma associação anatômica entre função serotonérgica e regulação de ritmos circadianos. ${ }^{82}$

Algumas avaliações do funcionamento do sistema serotonérgico através de estudos em plaquetas documentaram uma diminuição no número de sítios de captação de serotonina e de ligação da imipramina durante o ciclo menstrual em mulheres com SPM quando em comparação com mulheres controles, mesmo nos períodos assintomáticos. ${ }^{79}$ No entanto, outras medidas do funcionamento do sistema serotonérgico, como 
concentrações de cortisol após estímulo com clorofenilpiperazina, mostraram alterações somente no estado sintomático. ${ }^{83}$

Assim, é possível que flutuações na atividade serotonérgica durante o período pré-menstrual possam combinar-se com outras mudanças, tais como a dos hormônios gonadais (particularmente do estrógeno) e dos ritmos biológicos, contribuindo para o aparecimento de sintomas pré-menstruais em algumas mulheres.

\section{A vulnerabilidade aos sintomas pré-menstruais e implicações diagnósticas}

A vulnerabilidade e suscetibilidade a mudanças emocionais numa fase específica do ciclo menstrual há muito tempo é reconhecida. Tal aspecto pode ser exemplificado através da observação feita por E. von Feuchtersleben, em 1847, citado em um editorial assinado por Rubinow e Schmidt: ${ }^{84}$ “...em mulheres sensíveis, a menstruação é quase sempre caracterizada por inquietação mental, irritabilidade e tristeza". No entanto, ainda conforme o editorial, a pesquisa e identificação de fontes de vulnerabilidade em mulheres com síndrome pré-menstrual foi por longo tempo substituída pela busca de uma única explicação.

Essa situação tem sido modificada recentemente desde que a procura de alterações hormonais em mulheres com síndrome pré-menstrual tem redundado em resultados negativos, comprovando que o aparecimento dos sintomas independe de uma desregulação hormonal. ${ }^{85,86}$ Ao mesmo tempo, o envolvimento da fisiologia do ciclo menstrual tem sido substanciado pelas evidências de que a eliminação do ciclo ovariano pode resultar num alívio dos sintomas pré-menstruais. ${ }^{58}$ Mais ainda, há demonstrações recentes acerca do envolvimento dos hormônios ovarianos na gravidade dos sintomas pré-menstruais, ainda que esses mecanismos permaneçam especulativos. ${ }^{6}$

Diante desse panorama, cabe questionar a razão pela qual algumas mulheres respondem de maneira diferenciada ao que é essencialmente o mesmo estímulo - níveis normais de esteróides sexuais e sua flutuação - e exibem mudanças no humor em fases específicas do ciclo menstrual.

A partir dessa perspectiva, uma série de resultados tem sido reconsiderada como fatores que compõem o aspecto da vulnerabilidade, promovendo um contexto que determinaria a resposta à atuação de um agente precipitante, levando ao aparecimento de sintomas em mulheres predispostas à instabilidade do humor.

$\mathrm{Na}$ opinião de alguns pesquisadores ${ }^{77,79,87,88}$ vulnerabilidade poderia ser entendida como resultado da combinação entre mudanças decorrentes da flutuação hormonal e fatores e circunstâncias externas, os quais não são propriamente função do ciclo menstrual, mas que podem modular e influenciar a maneira como a mulher reagirá e administrará as mudanças ao longo do ciclo. Por exemplo, embora não seja um resultado consensual, uma associação entre piora dos sintomas pré-menstruais durante situações adversas ou experiências estressantes tem sido documentada em algumas investigações, condizendo com a experiência clínica de profissionais envolvidos com o atendimento e pesquisa da síndrome pré-menstrual. ${ }^{87,89}$
Expectativas negativas em relação à menstruação, aprendidas no ambiente social da mulher, também são citadas como um fator determinante na maneira como o ciclo menstrual é vivenciado. ${ }^{90}$ O quanto tal aprendizagem contribui para a manifestação de sintomas pré-menstruais ainda é uma questão em aberto. A importância de outros fatores extrínsecos ao ciclo menstrual na sintomatologia pré-menstrual também tem sido considerada. Algumas dimensões (características) específicas de personalidade, como "neuroticismo", são comumente mencionadas como fatores predisponentes e relevantes. ${ }^{35,91} \mathrm{~A}$ avaliação cognitiva através de testes neuropsicológicos, durante as fases folicular e lútea, mostrou que mulheres com SPM apresentaram dificuldades significativamente maiores em aprender material novo, mas esse resultado não foi dependente do período menstrual. ${ }^{92} \mathrm{O}$ fato de que estilos cognitivos influenciariam maneiras de conviver com adversidades ou eventos estressantes tem sido reconhecido e é motivo de tentativas esporádicas do tratamento da síndrome prémenstrual com o uso da terapia cognitiva. ${ }^{93-95}$

O contexto de vulnerabilidade também incluiria, entre os seus variados aspectos, a propensão a transtornos depressivos, produto de interações complexas entre fatores genéticos, de desenvolvimento e situacionais. Por exemplo, história de dificuldades emocionais, particularmente depressão, tem sido considerada como fator predisponente ao desenvolvimento de sintomas depressivos no período pré-menstrual, embora algumas investigações não tenham confirmado essas observações. ${ }^{28,33}$ Além disso, Graze e col. ${ }^{37}$ e Bancroft e col. ${ }^{96}$ aventaram que história passada de depressão poderia influenciar a gravidade e duração do humor depressivo peri-menstrual, desde que aquelas mulheres com história psiquiátrica prévia referissem humor deprimido mais acentuado, que persistisse durante a menstruação e, eventualmente, até alguns dias depois. A consistência desses resultados ainda está para ser determinada, pois, por motivos ignorados, Bancroft e Rennie ${ }^{97}$ não conseguiram chegar a iguais conclusões em estudo subseqüente.

Da mesma maneira, é possível especular que mulheres com traços específicos de vulnerabilidade teriam maior chance de responder com os mesmos sintomas a outras situações precipitantes, tais como mudanças hormonais abruptas. Ainda que matéria de debate, existem na literatura sugestões de que mulheres com disforia pré-menstrual também se apresentam freqüentemente sintomáticas durante outros períodos de instabilidade ou mudanças, como o período pós-parto e a menopausa. ${ }^{98}$ De fato, Arperls ${ }^{99}$ propõe que uma queda nos níveis cerebrais de estrógeno, além de um certo limiar com conseqüente redução nas concentrações de serotonina no Sistema Nervoso Central, seriam o fatores desencadeantes e denominadores comuns das alterações de humor dessas condições.

A questão da vulnerabilidade também se revela como uma explicação ao fato de que inúmeras variáveis biológicas, tais como ritmo da secreção de melatonina, temperatura corporal, secreção hormonal, e medidas do sistema serotonérgico e noradrenérgico, mostram-se alteradas tanto no período sintomático quanto assintomático do ciclo menstrual em mulheres com mudanças disfóricas na fase pré-menstrual. ${ }^{73,100,101}$

Assim, parece que a probabilidade de uma mulher apresentar 
síndrome pré-menstrual é determinada por uma série de fatores e por diversos domínios de risco. Os efeitos interativos desses fatores podem influenciar o quanto a mulher se ajusta às inúmeras mudanças fisiológicas que ocorrem ao longo do ciclo menstrual (tais como as hormonais, circadianas, e nos sistemas de neurotransmissão, particularmente serotonérgico e noradrenérgico), as quais modulariam o aparecimento de uma diversidade de sintomas durante o período pré-menstrual.

É provável que diferentes padrões de vulnerabilidade estejam correlacionados com diferentes manifestações sintomáticas, ou seja, em resposta ao mesmo agente precipitante, mulheres com vulnerabilidades diversas apresentariam sintomas também variados. Igualmente, flutuações no estado de vulnerabilidade responderiam pela considerável variação na apresentação dos sintomas, tanto na intensidade quanto no momento de aparecimento, que tem sido verificada em ciclos consecutivos.

Nesse sentido, embora uma associação entre disforia pré-menstrual e depressão seja assunto de interesse de longa data, só recentemente tem sido aventado o mesmo em relação aos transtornos ansiosos. Por exemplo, mulheres com disforia pré-menstrual também são mais propensas a desenvolver sintomas ansiosos em resposta à infusão de lactato ${ }^{102,103}$ e inalação de dióxido de carbono, ${ }^{104}$ estímulos considerados capazes de distinguir grupos de voluntários sadios de pacientes com transtorno do pânico.

Ainda que uma associação entre disforia pré-menstrual e transtornos ansiosos possa também ser considerada como comorbidade entre condições independentes, foi sugerido que mulheres com mudanças disfóricas associadas ao período prémenstrual teriam um traço de vulnerabilidade ao desenvolvimento de estados disfóricos, o qual se manifestaria como transtornos depressivos ou ansiosos. ${ }^{105}$ Assim, algumas delas seriam mais suscetíveis a desenvolver predominantemente sintomas ansiosos no período pré-menstrual e também, transtornos ansiosos ou do pânico, enquanto outras seriam mais vulneráveis a apresentar sintomas depressivos no pré-menstrual, e eventualmente transtorno depressivo. Dessa maneira, a natureza e a constelação dos sintomas estariam associados a particularidades da predisposição individual.

A comprovação dessas especulações e a integração de um número maior de evidências indicativas de que disforia prémenstrual compartilha características com os transtornos depressivos e ansiosos sugere que a proposta que consta no DSM-IV de caracterização dessa condição como uma entidade diagnóstica distinta e única de transtorno recorrente tem valor limitado e de pouca utilidade. Antes, sugere que a disforia pré-menstrual seria melhor conceituada como uma variação de entidades diagnósticas já existentes, mais do que como uma condição que necessita de uma nova rubrica diagnóstica.

Decorre disso o potencial benefício do estudo de alternativas à proposta diagnóstica atual. Entre outras sugestões, a consideração do padrão regular entre os sintomas e a fase lútea como um especificador de curso dos transtornos depressivos e possivelmente também dos transtornos ansiosos, a exemplo de outros que foram incorporadas no DSM-IV, tal como o transtorno de humor pós-parto (especifica início no pós parto).
Outras alternativas poderiam do mesmo modo ser investigadas. A compreensão da disforia pré-menstrual poderia ser ampliada também pelo exame de sua relação com as demais propostas de categorias diagnósticas incluídas no apêndice do DSM-IV, especificamente Transtorno Depressivo Breve Recorrente, ${ }^{106}$ cuja característica principal é a repetição de episódios breves de sintomas depressivos, semelhantes em número e gravidade aos observados nos episódios depressivos maiores, porém com menor duração (inferior a duas semanas). A descrição de uma síndrome denominada Ansiedade Breve Recorrente desponta como outra possibilidade de associação a ser futuramente explorada. ${ }^{107}$

\section{Referências bibliográficas}

1. Frank RT. The hormonal causes of premenstrual tension. Archives Neurology Psychiatry 1931;26:1053-7.

2. Greene R, Dalton K. The premenstrual syndrome. British Medical Journal 1953;1:1007-14

3. Munro A. Psychosomatic medicine. XI. Gynaecology. Practitioner 1972;209:713-20.

4. Suarez-Murias EL. The psychophysiologic syndrome of premenstrual tension with emphasis on the psychiatric aspect. Internat. Rec. Med. 1953;166:475-86.

5. Kramp JL. Studies on the premenstrual syndrome in relation to psychiatry. Acta Psychiatrica Scandinavica 1968;203:261-7.

6. Smith SL. Mood and the menstrual cycle. In: Greenblatt M, editor. Topics in Psychoendocrinology. Grune \& Stratton; 1975. p. 19-59.

7. American Psychiatric Association. Diagnostic and Statistical Manual of Mental Disorder. $3^{\text {rd }}$ ed. Revisada. Washington (DC): American Psychiatric Association; 1987.

8. Spitzer RL, Severino SK, Williams JBW, Parry BL. Late luteal phase dysphoric disorder and DSM-III-R. American Journal of Psychiatry 1989;146:892-7.

9. Hamilton JÁ, Gallant SJA. On a premenstrual psychiatric diagnosis: what's in a name? Prof. Psychol. Res. Pract. 1988;27:271-4.

10. American Psychiatric Association. DSM-IV Options Book: Work in Progress, Task Force on DSM-IV. Washington (DC): American Psychiatric Association; 1991.

11. American Psychiatric Association. Diagnostic and Statistical Manual of Mental Disorder. $4^{\text {th }}$ ed. Washington (DC): American Psychiatric Association; 1994.

12. Severino SK. Commentary: Late Luteal Phase Dysphoric Disorder - Disease or Dis-Ease? In: Gold JH, Severino SK, editors. Premenstrual Dysphorias: myths and realities. American Psychiatric Press; 1994a. p. 213-30.

13. Pires MLN, Calil HM. Transtorno Disfórico da Fase Lútea Tardia - uma nova categoria diagnóstica em psiquiatria? Revista ABP-APAL 1992;14(4):137-45.

14. Roy-Byrne PP, Hoban MC, Rubinow DR. The relationship of menstrually related mood disorders to psychiatric disorders. Clinical Obstetrics \& Gynecology 1987a;30:386-95.

15. Haskett RF, Steiner M, Osmun JN, Carroll BJ. Severe premenstrual tension: delineation of the syndrome. Biological Psychiatry 1980;15:121-39.

16. Halbreich U, Endicott J, Schacht S, Nee J. The diversity of premenstrual changes as reflected in the Premenstrual Assessment Form. Acta Psychiatrica Scandinavica 1982;65:46-65.

17. Mortola JF, Girton L, Yen SSC. Depressive episodes in premenstrual syndrome. American Journal of Obstetrics \& Gynecology 1989;161:16827.

18. Davidson JR, Miller RD, Turnbull CD, Sullivan JL. Atypical depression. Archives of General Psychiatry 1982;39:527-34.

19. Freeman EW, Rickels K, Schweizer E, Ting T. Relationships between age and symptom severity among women seeking medical treatment for premenstrual symptoms. Psychological Medicine 1995a;25:309-15.

20. Hamilton M. Development of a rating scale for primary depressive illness. British Journal of Social \& Clinical Psychology 1967;6:278-96.

21. Mcgrath PJ, Stewart JW, Harrison WM, Ocepek-Welikson K, Rabkin JG, Nunes EN, et al. Predictive value of symptoms of atypical depression for differential drug treatment outcome. Journal of Clinical Psychopharmacology 1992;12:197-202.

22. Beck AT, Ward CH, Mendelsohn M, Mock J, Erbaugh J. An inventory for 
measuring depression. Archives of General Psychiatry 1961;4:561-71.

23. Christensen AP, Oei TPS. Correlates of premenstrual dysphoria in helpseeking women. Journal of Affective Disorders 1995a;33:47-55.

24. Chan AF, Mortola JF, Wood SH, Yen SSC. Persistence of premenstrual syndrome during low-dose administration of the progesterone antagonist RU 486. Obstetrics \& Gynecology 1994;84:1001-5.

25. Schmidt PJ, Purdy RH, Moore-Jr PH, Paul SM, Rubinow DR. Circulating levels of anxiolytic steroids in the luteal phase in women with premenstrual syndrome and in control subjects. Journal of Clinical Endocrinology \& Metabolism 1994;79:1256-60.

26. Frank E, Carpenter LL, Kupfer DJ. Sex differences in recurrent depression: are there any that are significant? American Journal of Psychiatry 1988;145:41-5.

27. Spitzer RL, Endicott J, Robins E. Research diagnostic criteria: rationale and reliability. Archives of General Psychiatry 1978;35:773-82.

28. Hurt SW, Schnurr PP, Severino SK, Freeman EW, Gise LH, Rivera-Rovar A, et al. Late luteal phase dysphoric disorder in 670 women evaluated for premenstrual complaints. American Journal of Psychiatry 1992;149:525-30.

29. Roy-Byrne PP, Rubinow DR, Hoban MC, Parry BL, Rosenthal NE, Nurnberger JI, et al. Premenstrual changes: a comparison of five populations. Psychiatry Research 1986a; 17:77-85.

30. Dye L, Warner P, Bancroft J. Food craving during the menstrual cycle and its relationship to stress, happiness of relationship and depression; a preliminary enquiry. Journal of Affective Disorder 1995;34:157-64.

31. Hill AJ, Heaton-Brown L. The experience of food craving: a prospective investigation in healthy women. Journal of Psychosomatic Research 1994;38:801-14.

32. Halbreich U, Endicott J. Relationship of dysphoric premenstrual changes to depressive disorders. Acta Psychiatrica Scandinavica 1985;71:331-8.

33. Endicott J. The menstrual cycle and mood disorders. Journal of Affective Disorder 1993;29:193-200.

34. Regier DA, Burke-Jr JD, Burke KC. Comorbidity of affective and anxiety disorders in the NIMH Epidemiologic Catchment Area Program. In: Maser JD, Cloninger CR, editors. Comorbidity of mood and anxiety disorders. Washington (DC): American Psychiatric Press; 1990. p. 113-22.

35. Merikangas KR, Foeldenyi M, Angst J. The Zurich Study: XIX. Patterns of menstrual disturbances in the community: results of the Zurich Cohort Study. European Archives of Psychiatry \& Clinical Neuroscience 1993;243:23-32.

36. American Psychiatric Association. Diagnostic and Statistical Manual of Mental Disorder. $3^{\text {rd }}$ ed. Washington (DC): American Psychiatric Association; 1980.

37. Graze KK, Nee J, Endicott J. Premenstrual depression predicts future major depressive disorder. Acta Psychiatrica Scandinavica 1990;81:201-5.

38. Rubinow DR, Schmidt PJ. Models for the development and expression of symptoms in premenstrual syndrome. Psychiatric Clinics of North America 1989;12:53-68.

39. Roy-Byrne P, Rubinow DR, Gold PW, Post RM. Possible antidepressant effect of oral contraceptives: case report. Journal of Clinical Psychiatry 1984;45:350-2

40. Ploeg van der HM, Lodder EB. Longitudinal measurement in the diagnostics of the premenstrual syndrome. Journal of Psychosomatic Research 1993;37:33-8.

41. Metcalf MG, Braiden V, Livesey JH. Symptom cyclicity in women with premenstrual syndrome: an 8-year follow-up study. Journal of Psychosomatic Research 1992;36:237-41.

42. Endicott J. Differential Diagnoses and Comorbidity. In: Gold JH, Severino SK, editors. Premenstrual Dysphorias: myths and realities. American Psychiatric Press; 1994. p. 3-17.

43. Harrison WM, Endicott J, Nee J. Treatment of premenstrual depression with nortriptyline: a pilot study. Journal of clinical Psychiatry 1989a;50:136-9.

44. Harrison WM, Endicott J, Nee J, Glick H, Rabkin JG. Characteristics of women seeking treatment for premenstrual syndrome. Psychosomatics 1989b;30:405-41.

45. Freeman E, Rickels K, Sondheimer SJ, Polansky M. Ineffectiveness of progesterone suppository treatment for premenstrual syndrome. JAMA 1990;264:349-53.

46. Kantero RL, Widholm O. Correlations of menstrual traits between adolescent girls and their mothers. Acta Obstetricia et Gynecologica Scandinavica 1971;14(supl.14):30-6.

47. Glick H, Endicott J, Nee J. Premenstrual changes: are they familial? Acta Psychiatrica Scandinavica 1993;88:149-55.

48. Endicott J, Halbreich U. Retrospective report of premenstrual depressive changes: factor affecting confirmation by daily ratings. Psychopharmacology Bulletin 1982;18:109-12.
49. Dalton K, Dalton ME, Guthriek K. Incidence of the premenstrual syndrome in twins. British Medical Journal Clinical Research Ed. 1987;295:1027-8.

50. Kendler KS, Silberg JL, Neale MC, Kessler RC, Heath AC, Eaves LJ. Genetic and environmental factors in the aetiology of menstrual, premenstrual and neurotic symptoms: a population-based twin study. Psychological Medicine 1992;22:85-100

51. Condon JT. The premenstrual syndrome: a twin study. British Journal of Psychiatry 1993;162:481-6.

52. Steiner M, Haskett RF, Carroll BJ. Premenstrual tension syndrome: the development of research diagnostic criteria and new rating scales. Acta Psychiatrica Scandinavica 1980;62:177-90.

53. Rausch JL, Janowsky DS, Risch C, Judd LL, Huey LY. Hormonal and neurotransmitter hypotheses of premenstrual tension. Psychopharmacology Bulletin 1982;18:26-34.

54. Bäckström T, Sanders D, Leask R, Davidson D, Warner P, Bancroft J. Mood, sexuality, hormones, and the menstrual cycle. II. Hormone levels and their relationship to the premenstrual syndrome. Psychosomatic Medicine 1983;45:503-7.

55. Rubinow DR, Hoban C, Grover GN, Galloway DS, Roy-Byrne P, Andersen $\mathrm{R}$, et al. Changes in plasma hormones across the menstrual cycle in patients with menstrually related mood disorder and in control subjects. American Journal of Obstetrics \& Gynecology 1988;158:5-11.

56. Parry BL, Gerner RH, Wilkins JN, Halaris AE, Carlson HE, Hershman JM, et al. CSF and endocrine studies of premenstrual syndrome. Neuropsychopharmacology 1991;5:127-37.

57. Schmidt PJ, Nieman LK, Grover GN, Muller KL, Merriam GR, Rubinow DR. Lack of effect of induced menses on symptoms in women with premenstrual syndrome. New England Journal of Medicine 1991;324:1174-9.

58. Severino SK, Moline ML. Premenstrual syndrome. Identification and management. Drugs 1995;49:71-82.

59. Smith RNJ, Studd JW, Zamblera D, Holland EFN. A randomised comparison over 8 months of $100 \mathrm{mg}$ and $200 \mathrm{mg}$ twice weekly doses of transdermal oestradiol in the treatment of severe premenstrual syndrome. British Journal of Obstetrics and Gynaecology 1995;102:475-84.

60. Schmidt PJ, Nieman LK, Danaceau MA, Adams LF, Rubinow DR. Differential behavioral effects of gonodal steroids in women with and in those without premenstrual syndrome. New England Journal of Medicine 1998;338:209-16.

61. Redei E, Freeman EW. Daily plasma estradiol and progesterone levels over the menstrual cycle and their relation to premenstrual symptoms. Psychoneuroendocrinology 1995;20:259-67.

62. Hammarbäck S, Damber JE, Bäckström T. Relationship between symptoms severity and hormone changes in women with premenstrual syndrome. Jounal of Clinical Endocrinology \& Metabolism 1989;68:125-30.

63. Holsboer F. Neuroendocrinology of mood disorders. In: Bloom FE, Kupfer DJ, editors. Psychopharmacology: the fourth generation of progress. New York: Raven Press Ltd; 1995. p. 957-69.

64. Roy-Byrne PP, Rubinow DR, Gwirtsman H, Hoban MC, Grover GN. Cortisol response to dexamethasone in women with premenstrual syndrome. Neuropsychobiology 1986b;16:61-3.

65. Rabin DS, Schmidt PJ, Campbell G, Gold PW, Jensvold M, Rubinow DR, et al. Hypothalamic-pituitary-adrenal function in patients with premenstrual syndrome. Journal of Clinical Endocrinology \& Metabolism 1990;71:1158-62.

66. Schmidt PJ, Grover GN, Roy-Byrne PP, Rubinow DR. Thyroid function in women with premenstrual syndrome. Journal of Clinical Endocrinology \& Metabolism 1993;76:671-4

67. Roy-Byrne PP, Rubinow DR, Hoban MC, Grover GN, Blank D. TSH and prolactin responses to TRH in patients with premenstrual syndrome. American Journal of .Psychiatry 1987b;144:480-4.

68. Nikolai TF, Mulligan GM, Gribble RK, Harkins PG, Meier PR, Roberts RC. Thyroid function and treatment in premenstrual syndrome. Journal of Clinical Endocrinology \& Metabolism 1990;70:1108-13

69. Anderson JL, Wirz-Justice A. Biological rhythms in the pathophysiology and treatment of affective disorders. In: Horton R, Katona C, editors. Biological Aspects of Affective Disorders. Academic Press; 1991. p. 223-69.

70. Wehr TA, Wirz-Justice A, Goodwin FK, Duncan W, Gillin JC. Phase advance of the circadian sleep wake cycle as an antidepressant. Science 1979;206:710-3.

71. Parry BL, Cover H, Mostofi N, Leveau B, Sependa PA, Resnick A, et al. Early versus late partial sleep deprivation in patients with premenstrual dysphoric disorder and normal comparison subjects. American Journal of Psychiatry 1995;152:404-12. 
72. Pires MLN. Disforia pré-menstrual: evolução do conceito, dificuldades diagnósticas e aspectos terapêuticos - efeitos da privação parcial de sono [ tese - Doutorado]. São Paulo: Universidade Federal de São Paulo, Escola Paulista de Medicina; 1996.

73. Parry B, Berga SC, Kripke DF, Gillin JC. Melatonin, light therapy, and premenstrual syndrome. In: Shafii M, Shafii SL, editors. Biological rhythms, mood disorders, light therapy, and the pineal gland. American Psychiatric Press; 1990. p.177-189.

74. Parry BL, Hauger R, Lin E, Leveau B, Mostofi N, Clopton P.L, et al. Neuroendocrine effects of light therapy in late luteal phase dysphoric disorder. Biological Psychiatry 1994;36:356:64.

75. Lee KA, Shaver JF, Giblin EC, Woods NF. Sleep patterns related to menstrual cycle phase and premenstrual affective symptoms. Sleep 1990;13:403-9.

76. Parry BL, Mendelson WB, Duncan WC, Sack DA, Wehr TA. Longitudinal sleep EEG, temperature, and activity measurements across the menstrual cycle in patients with premenstrual depression and in age-matched controls. Psychiatry Research 1989;30:285-303.

77. Severino SK. Late luteal phase dysphoric disorder: a scientific puzzle. Medical Hypotheses 1993;41:229-34.

78. Leibenluft E. Do gonadal steroids regulate circadian rhythms in humans? Journal of Affective Disorder 1993;29:175-81.

79. Halbreich U, Tworek H. Altered serotonergic activity in women with dysphoric premenstrual syndromes. International Journal of Psychiatry in Medicine 1993;23:1-27.

80. Severino SK. A Focus on 5-hydroxytryptamine (serotonin) and psychopatology. In: Gold JH, Severino SK, editors. Premenstrual Dysphorias: myths and realities. American Psychiatric Press; 1994b. p. 67-98.

81. Sumner BEH, Fink G. Estrogen increases the density of 5-hydroxytryptamine $\left(2^{\mathrm{a}}\right)$ receptors in cerebral cortex and nucleus accumbens in the female rat. Journal of Steroid Biochemistry \& Molecular Biology 1995;54:15-20

82. Parnavelas JG, Dinopoulos A, Davies SW. The central visual pathways. In: Björklund A, Hökfelt T, Swanson LW, editors. Handbook of Chemical Neuroanatomy. Elsevier; 1989. p. 44.

83. Halbreich U. Gonodal hormones and antihormones, serotonin and mood. Psychopharmacology Bulletin 1990;26:291-5

84. Rubinow DR, Schmidt PJ. The treatment of premenstrual syndrome. Forward into the past. New England Journal of Medicine 1995;332:1574-5.

85. Rubinow DR, Schmidt PJ. Premenstrual syndrome: a review of endocrine studies. The Endocrinologist 1992;2:47-54.

86. Nunes MG. Síndrome pré-menstrual: aspectos metabólicos e hormonais [Tese - Mestrado]. São Paulo: Universidade Federal de São Paulo, Escola Paulista de Medicina; 1996.

87. Bancroft J. The premenstrual syndrome - a reappraisal of the concept and the evidence. Psychological Medicine 1993;(supl.24):1-47.

88. Halbreich U. Menstrually related disorders: what we do know, what we only believe that we know, and what we know that we do not know. Critical Review in Neurobiology 1995a;9:163-175.

89. Gise LH, Lebovits AH, Paddison PL, Strain JJ. Issues in the identification of premenstrual syndromes. Journal of Nervous \& Mental Disease 1990;178:228-34.

90. Dan AJ, Monagle 1. Sociocultural influences on women's experiences of perimenstrual symptoms. In: Gold JH, Severino SK, editors. Premenstrual Dysphorias: myths and realities. American Psychiatric Press; 1994. p. 201-11.
91. Freeman EW, Schweizer E, Rickels K. Personality factors in women with premenstrual syndrome. Psychosomatic Medicine 1995b;57:453-9.

92. Keenan PA, Stern RA, Janowsky DS, Pedersen CA. Psychological aspects of premenstrual syndrome I: cognition and memory. Psychoneuroendocrinology 1992; 17:179-87.

93. Morse CA, Dennerstein L. Cognitive therapy for premenstrual syndrome. In: Brush MG, Goudsmit EM, editors. Functional disorders of the menstrual cycle. John Wiley \& Sons Ltd; 1988. p. 177-90.

94. Christensen AP, Oei TP. The efficacy of cognitive behaviour therapy in treating premenstrual dysphoric changes. Journal of Affective Disorder 1995b;33:57-63.

95. Blake F, Salkovski P, Gath D, Day A, Garrod A. Cognitive therapy for premenstrual syndrome: a controlled trial. Journal of Psychosomatic Research 1998;45:307-18

96. Bancroft J, Rennie D, Warner P. Vulnerability to perimenstrual mood change: the relevance of a past hystory of depressive disorder. Psychosomatic Medicine 1994;56:225-31.

97. Bancroft J, Rennie D. Perimenstrual depression: its relationship to pain, bleeding, and previous history of depression. Psychosomatic Medicine 1995;57:445-52

98. Hamilton JA, Parry BL, Blumenthal SJ. The menstrual cycle in context. I: Affective syndromes associated with reproductive hormonal changes. Journal of Clinical Psychiatry, 1988;49:474-80.

99. Arpels JC. The female brain hypoestrogenic continnum from the premenstrual syndrome to menopause. Journal of Reproductive Medicine 1996;41:633-9.

100. Severino SK, Wagner DR, Moline ML, Hurt SW, Pollak CP, Zendell S. High nocturnal body temperature in premenstrual syndrome and late luteal phase dysphoric disorder. American Journal of Psychiatry 1991;148:1329-35.

101. Yatham LN. Is 5 htla receptor subsensitivity a trait marker for late luteal phase dysphoric disorder? A pilot study. Canadian Journal of Psychiatry revue Canadienne de Psychiatrie 1993;38:662-4.

102. Facchinetti F, Romano G, Fava M, Genazzani AR. Lactate infusion induces panic attacks in patients with premenstrual syndrome. Psychosomatic Medicine 1992;54:288-96.

103. Sandberg D, Endicott J, Harrison W, Nee J, Gorman J. Sodium lactate infusion in late luteal phase dysphoric disorder. Psychiatry Research 1993;46:79-88.

104. Harrison WM, Sandberg D, Gorman JM, Fyer M, Nee J, Uy J, et al. Provocation of panic with carbon dioxide inhalation in patients with premenstrual dysphoria. Psychiatry Research 1989c;27:183-92.

105. Halbreich U. Premenstrual dysphoric disorders, anxiety, and depressions: vulnerability traits or comorbidity. Archives of General Psychiatry 1995b;52:606 [letter].

106. Angst J, Merikangas K, Scheidegger P, Wicki W. Recurrent brief depression: a new subtype of affective disorder. Journal of Affective Disorder 1990;19:87-98.

107. Angst J, Wicki W. The Zurich study. XIII. Recurrent brief anxiety. European Archives of Psychiatry \& Clinical Neuroscience 1992;241:296-300.

\section{Correspondência}

Maria Laura Nogueira Pires / Helena Maria Calil

Rua Botucatu, $862-1^{\circ}$ andar

CEP 04023-900, São Paulo - SP 